\title{
NUEVOS RELEVAMIENTOS DE ESTRUCTURAS LÍTICAS Y PIE- DRAS PARADAS EN LAS NACIENTES DEL RIO SAUCE GRANDE (PARTIDO DE TORNQUIST, PROVINCIA DE BUENOS AIRES).
}

\section{SURVEY OF THE LYTIC STRUCTURES AND STANDING STONES IN THE HEADWATERS OF THE SAUCE GRANDE RIVER (TORNQUIST, BUENOS AIRES PROVINCE)}

\author{
María Cecilia Panizza ${ }^{1}$, Fernando Oliva ${ }^{2}$, Anabella Sfeir $^{3}$, Camila Oliva ${ }^{4}$ y Gimena \\ Devoto $^{5}$ \\ ${ }^{1}$ Becaria CONICET - CEAR (FHumyAr -UNR), E-mail: mcpanizza@yahoo.com.ar \\ ${ }^{2}$ CEAR (FHumyAr -UNR), FCNyM (UNLP), y DPPC (ICPBA), E-mail: fwoliva@unr.edu.ar; \\ ${ }^{3}$ Becaria CIN - FCNyM (UNLP), CEAR (FHumyAr -UNR), E-mail: anabellasfeir@yahoo.com.ar \\ ${ }^{4}$ Becaria CIN - FCNyM (UNLP), CEAR (FHumyAr -UNR), E-mail: oliva.cami@gmail.com \\ ${ }^{5}$ Becaria CIN - FCNyM (UNLP), CEAR (FHumyAr -UNR), E-mail:gimenadevoto@outlook.com
}

Presentado el: 18/02/2013 - Aceptado 15/04/2013

\section{Introducción}

Dentro del conjunto de evidencias arqueológicas documentadas para el Sistema Serrano de Ventania, se destacan por su número, los sitios arqueológicos que presentan estructuras líticas y/o piedras paradas. Los estudios de este tipo de registro se inician tardíamente en la década del ‘90 (Madrid 1991a, 1991b; Oliva y Roa 1998, Roa y Saghessi 2004) profundizándose la sistematización de los mismos en el último lustro dado el riesgo inminente de deterioro y alteración al que se ven expuestos (Devoto et al. 2012, Oliva 2011, Oliva y Panizza 2010, 2012; Sfeir y Oliva 2012). Los datos obtenidos permitieron establecer su área de ocurrencia fundamentalmente en la zona centrooriental, sector que corresponde a los cordones serranos de la Ventana y de Pillahuinco. Hasta el momento, se considera que el conjunto de piedras paradas y/o pircados fueron construidas por grupos humanos indígenas sin mayores precisiones de su inicio, hasta momentos en donde es posible atribuirlas a ocupaciones localizadas a fines del Holoceno (Oliva y Panizza 2012). Se considera que las sociedades indígenas utilizaron en todos los casos materias primas locales procedentes de las inmediaciones donde se localizan los sitios, de los cordones Esmeralda y de la Ventana, cuya litología está integrada por areniscas de grano grueso hasta conglomerádico, por areniscas cuarcíticas muy homogéneas de grano fino a muy fino, compactas, macizas y densas; por areniscas esquistosas de grano fino entre las que se intercalan bancos de esquistos arcillosos, y 
por metaarenitas de cuarzo-feldespato-muscovita estratificadas y bancos delgados de filitas y pizarras (Harrington 1947). Durante el trabajo de campo se observaron indicios de la extracción de estas materias primas por explotación contemporánea, provocando acumulaciones de lajas y rocas voluminosas en las cercanías de los sitios arqueológicos descriptos en este trabajo.

El Sistema Serrano de Ventania está localizado en el sector sur del Área Ecotonal Húmedo Seca Pampeana (AEHSP), y es una de las tres serranías que sobresalen en el paisaje de llanuras que caracteriza a la Región Pampeana. Esta zona de ecotono posee una variedad y diversidad de recursos que combina elementos de la Pampa Húmeda y Seca, y de Nordpatagonia, lo cual constituyó un espacio atractivo para los grupos humanos que lo habitaron, que obtenían en este ambiente agua potable, presas animales y plantas comestibles, materia prima para sus herramientas, entre otros beneficios.

En este trabajo se presentan los hallazgos vinculados a relevamientos de estructuras líticas y piedras paradas realizados en el partido de Tornquist durante el mes de agosto de 2012. El equipo de investigación accedió a diversos predios rurales próximos a la localidad de Villa Ventana, con el fin de prospectar los sitios arqueológicos, en el marco de proyectos de investigación acreditados en las Universidades Nacionales de La Plata y Rosario.

\section{Materiales y Métodos}

El relevamiento realizado en las nacientes del Río Sauce Grande, consistió en el registro de datos vinculados con los sitios arqueológicos como su ubicación geográfica y visibilidad en el terreno, toma de medidas de las estructuras y piedras (alto, ancho, espesor, orientación respecto al norte), y su relación con rasgos relevantes del paisaje circundante (cercanía a cursos de agua, distancia a otros sitios arqueológicos, entre otros), además de la evaluación del grado de conservación y la acción de los agentes de deterioro, como el clima, la vegetación, los animales, e incluso la actividad humana. Particularmente cabe mencionar que se documentó fotográficamente las evidencias arqueológicas y el contexto espacial, para lo cual se realizaron croquis de la disposición de las estructuras de piedras en vinculación a otros registros arqueológicos y naturales. En este trabajo se presentan los resultados de las prospecciones de los establecimientos, la identificación de los sitios y la recolección de datos. Estas tareas de registro del patrimonio arqueológico monumental dieron como resultado la determinación de un total de 13 sitios (Figura 1), cuyas características se sintetizan en la Tabla 1.

\section{Localidad arqueológica Las Vertientes}

Ubicada en la estancia Las Vertientes, contigua a la ruta 76, y frente al Parque Provincial E. Tornquist. En el área próxima a la tranquera de acceso, recientemente arada, se identificó un sitio de superficie que presentaba abundante cantidad de materiales líticos dispersos, realizados sobre materias primas locales y con diferentes grados de formatización. Hacia el interior del predio, en una zona donde se observa un monte en su mayor parte constituido por álamos, se relevaron cuatro sitios arqueológicos, entre los que se destacaban una estructura de forma de " $U$ " adosada, y tres alineamientos de piedras paradas, uno de los cuales está localizado en el faldeo de un cerro próximo (alcanzando una altitud de unos 550 metros sobre el nivel del mar). El conjunto del sitio 


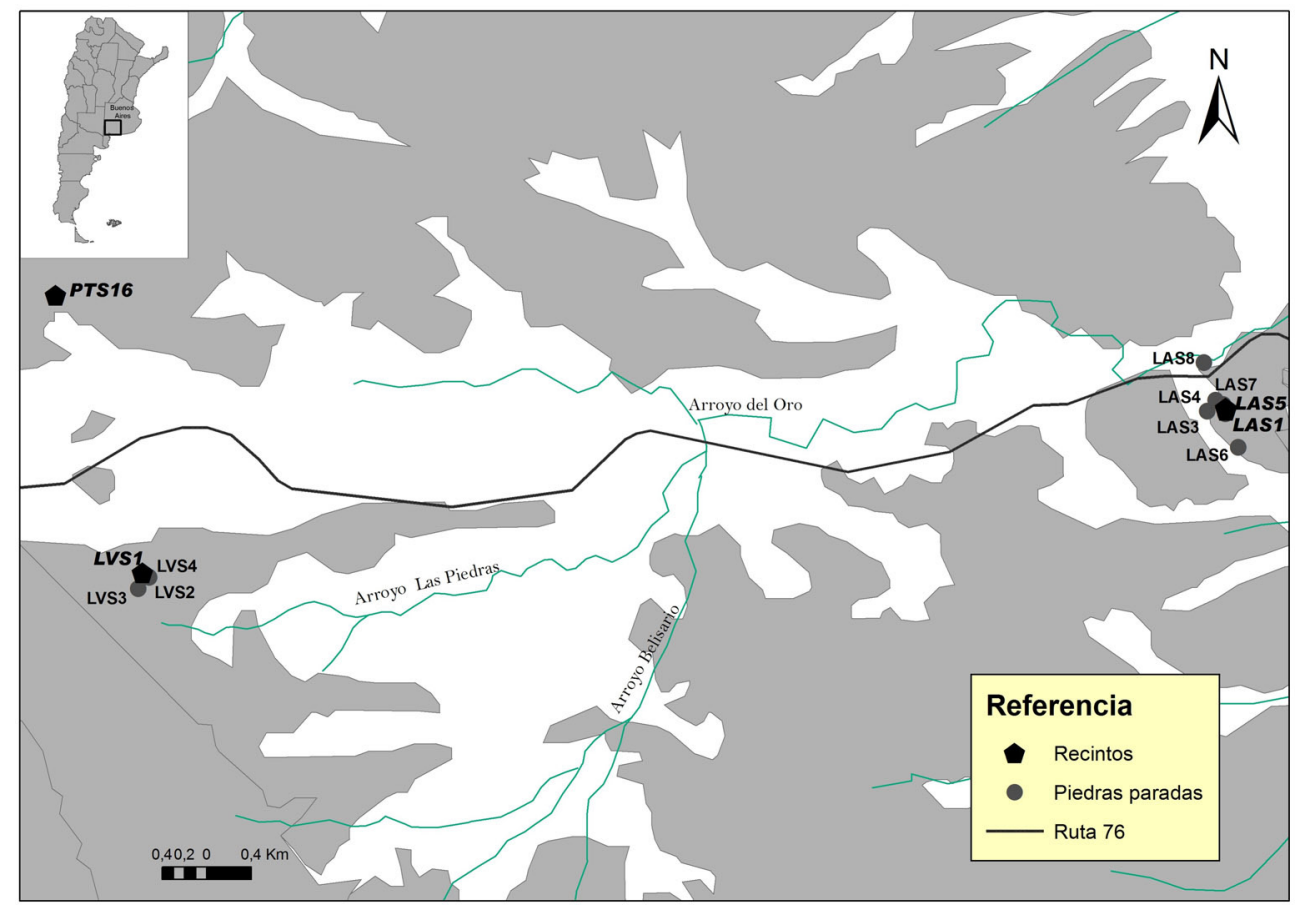

Figura 1. Mapa de las nacientes del río Sauce Grande donde se ubican las estructuras líticas y las piedras paradas relevadas.
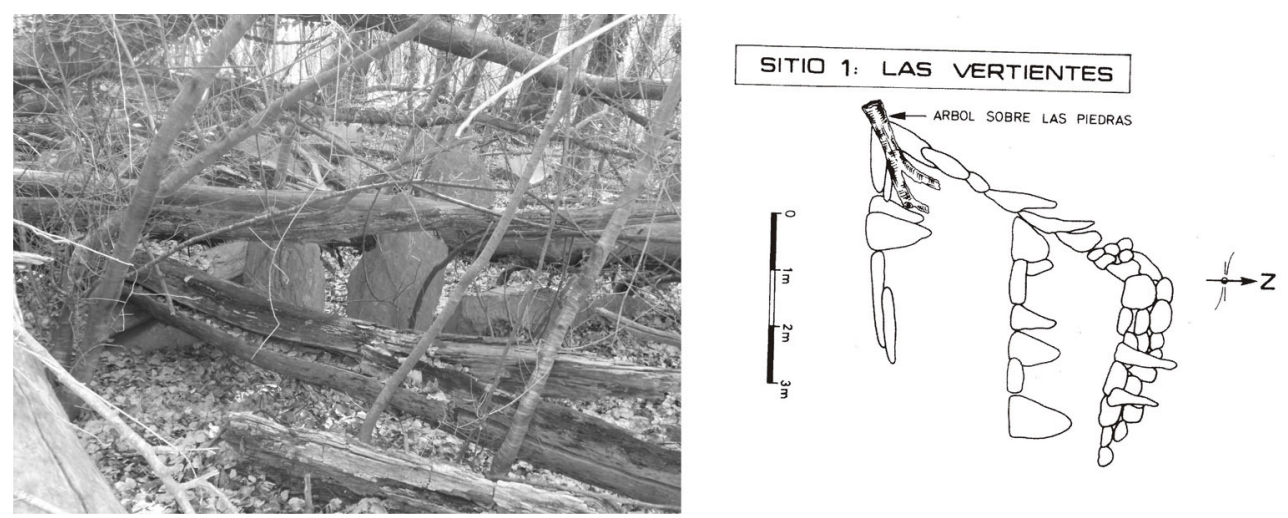

Figura 2. Recinto de Las Vertientes Sitio 1, fuertemente afectado por la abundante cubierta vegetal.

1 constituye una figura rectangular de 6,5 $\mathrm{m}$ de largo por 5,5 $\mathrm{m}$ de ancho, con piedras que varían de $1,5 \mathrm{~m}$ a $0,75 \mathrm{~m}$. Se documentó una fuerte acción de deterioro sobre este conjunto monumental, producto principalmente de la abundante cobertura vegetal que también obstaculiza su visualización (Figura 2). En forma simultánea se verificó la presencia de alambres y vidrios sobre las evidencias arqueológicas o próximas a ellas lo cual junto al monte constituido por especies vegetales no autóctonas indican la actividad de pobladores recientes como causa principal de deterioro de los sitios. 


\begin{tabular}{|c|c|c|c|c|}
\hline SITIO & ALTITUD & $\begin{array}{l}\text { DISTANCIA A } \\
\text { CURSOSDE AGUA }\end{array}$ & TIPO DE ESTRUCTURA & Nro, por SITIO \\
\hline Las Acacias Sitio 1 & $321 \mathrm{msnm}$ & entre 50 y $80 \mathrm{~m}$ & $\begin{array}{l}\text { recinto cerrado de piedras } \\
\text { clavadas de forma } \\
\text { rectangular }\end{array}$ & 1 \\
\hline Las Acacias Sitio 2 & $321 \mathrm{msnm}$ & entre 100 y $150 \mathrm{~m}$ & piedras paradas & 1 \\
\hline Las Acacias Sitio 3 & $336 \mathrm{msnm}$ & entre 200 y $300 \mathrm{~m}$ & piedras paradas & 2 \\
\hline Las Acacias Sitio 4 & $326 \mathrm{msnm}$ & entre 100 y $150 \mathrm{~m}$ & piedras paradas & 1 \\
\hline Las Acacias Sitio 5 & $323 \mathrm{msnm}$ & entre $50 \mathrm{y} 80 \mathrm{~m}$ & $\begin{array}{l}\text { recinto cerrado de ladrillos de } \\
\text { forma cuadrangular }\end{array}$ & 1 \\
\hline Las Acacias Sitio 6 & $327 \mathrm{msnm}$ & $\mathrm{Om}$ & piedra parada & 1 \\
\hline Las Acacias Sitio 7 & $324 \mathrm{msnm}$ & entre $50 \mathrm{y} 80 \mathrm{~m}$ & piedra parada & 1 \\
\hline Las Acacias Sitio 8 & $328 \mathrm{msnm}$ & entre 10 y $50 \mathrm{~m}$ & piedra parada & 1 \\
\hline Las Vertientes Sitio 1 & $330 \mathrm{msnm}$ & entre 10 y $50 \mathrm{~m}$ & $\begin{array}{l}\text { recinto abierto de piedras } \\
\text { clavadas y acumuladas con } \\
\text { forma de } 2 \text { U adosadas }\end{array}$ & 1 \\
\hline Las Vertientes Sitio 2 & $500 \mathrm{msnm}$ & entre $50 \mathrm{y} 80 \mathrm{~m}$ & piedra parada & 12 \\
\hline Las Vertientes Sitio 3 & $500 \mathrm{msnm}$ & menos de $10 \mathrm{~m}$ & piedras paradas & 18 \\
\hline Las Vertientes Sitio 4 & $480 \mathrm{msnm}$ & menos de $10 \mathrm{~m}$ & piedras paradas & 4 \\
\hline Parque Tornquist Sitio 10 & $485 \mathrm{msnm}$ & entre 50 y $80 \mathrm{~m}$ & $\begin{array}{l}\text { recinto abierto de piedras } \\
\text { acumuladas de forma sub- } \\
\text { circular }\end{array}$ & 1 \\
\hline
\end{tabular}

Tabla 1. Características de los sitios arqueológicos relevados.

\section{Localidad arqueológica Las Acacias}

En el establecimiento Las Acacias se relevaron 8 sitios arqueológicos de estructuras líticas y piedras paradas en diversos sectores del terreno, entre los cuales destaca una estructura de forma cuadrangular de aproximadamente $70 \mathrm{~m}^{2}$, compuesta por piedras clavadas, y que presenta uno de sus lados lindando con un alambrado contemporáneo (Figura 3). Varias de las piedras que constituyen este "muro" registran la presencia de líquenes, otra de las piedras presenta pelos de ganado adheridos a su superficie, y en todo el terreno correspondiente al sitio se documentaron numerosos hoyos realizados por roedores. Por otra parte, en el centro de esta estructura se encuentra otra piedra clavada de mayor altura $(1,96 \mathrm{~m})$ sobre el terreno, la cual -dada sus características y comentarios de algunos pobladores locales- habría sido colocada en ese lugar en tiempos recientes, con posterioridad a la construcción de la estructura circundante, con el fin de resignificar el sitio arqueológico vinculado a la actividad turística. Otra acción importante de deterioro realizada sobre este sitio es un pozo de unos $9 \mathrm{~m} 2$ localizado en el sector noreste, producto de una excavación furtiva. A unos $50 \mathrm{~m}$ se observa otro sitio con piedras acumuladas o pilas de rocas, con evidencias de haber sido alterado estructuralmente, y que presenta una estructura de metal rodeándolo, con el fin aparente de protegerlo. En las inmediaciones se detectaron cimientos de ladrillos correspondientes a momentos históricos recientes (sitio 5), y vestigios de actividad lajera. Además, una piedra parada fue registrada al otro lado de la ruta (sitio 8), siguiendo el eje de las otras evidencias monumentales relevadas, lo que nos 


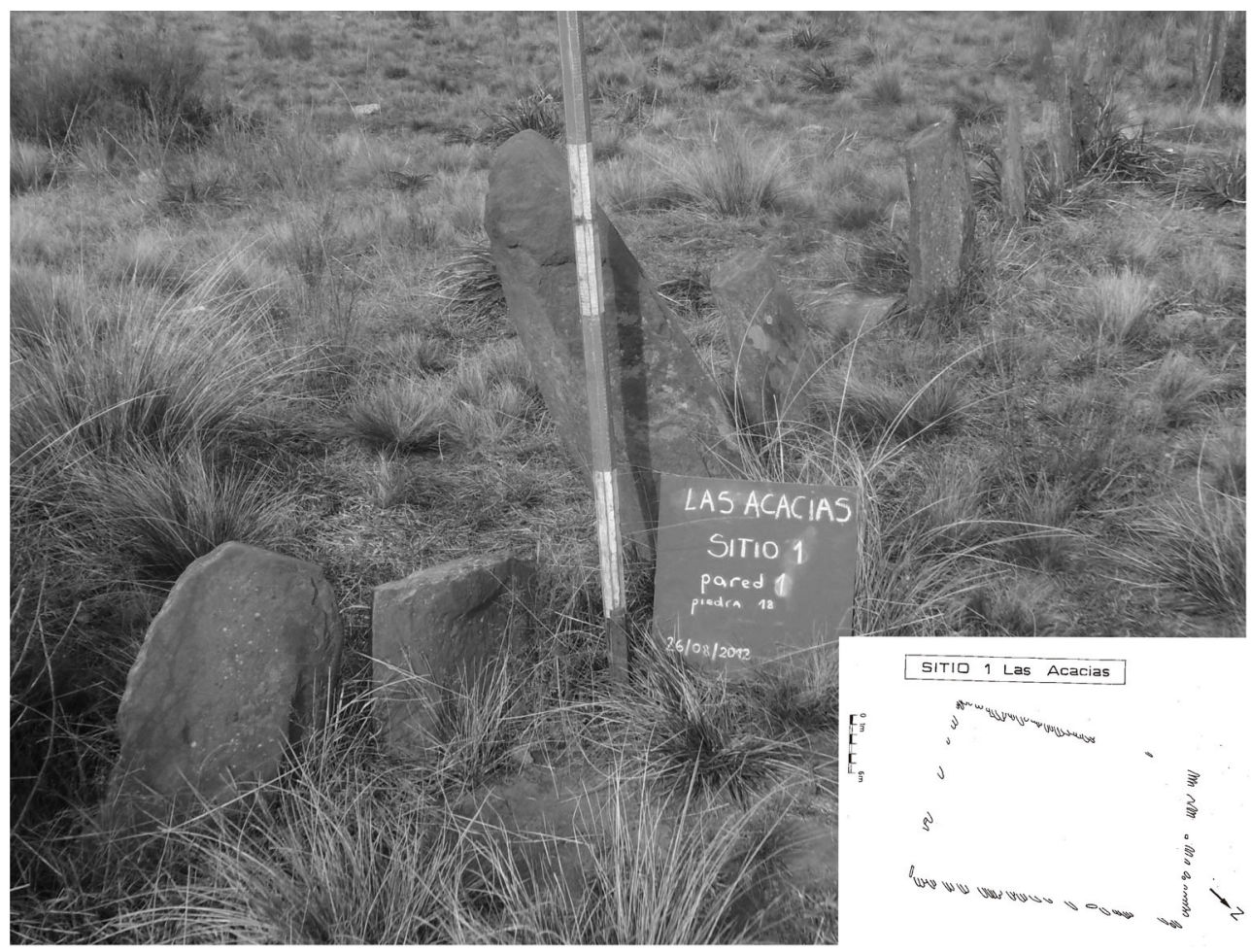

Figura 3. Las Acacias Sitio 1.

indica una continuidad en la ocupación del espacio, que fue altamente deteriorado en el momento de la construcción de la carretera.

\section{Parque Tornquist sitio 10}

En el Parque Provincial Ernesto Tornquist, se han identificado hasta el momento cuevas con arte rupestre, sitios superficiales y con estructuras de piedras y piedras paradas. En esta oportunidad se informa sobre el sitio 10, el cual se localiza en el sector de entrada al área de reserva del Parque en proximidades del camino interior. Este sitio está constituido por dos pircados paralelos entre sí con forma subcircular y una longitud máxima de $29 \mathrm{~m}$, espesor de las paredes de $55 \mathrm{~cm}$ en promedio y la altura varía entre 27 y $75 \mathrm{~cm}$. Las paredes que forman la curva dejan un espacio interior de unos $8 \mathrm{~m}$ promedio (Figura 4). Se encuentra a unos $60 \mathrm{~m}$ aproximadamente en línea recta al curso de agua, siendo su estado de conservación bueno, aunque está afectado por líquenes y pastizales en un porcentaje que no supera el $20 \%$. Cabe destacar que este sitio utiliza el afloramiento natural en uno de sus sectores como parte constitutiva de la estructura.

En años anteriores, otros sitios arqueológicos tanto de estructuras de piedras como de piedras paradas ya habían sido documentados en otros sectores del Parque Provincial (Oliva y Panizza 2012), principalmente en las proximidades al arroyo del Oro (i.e. sitios Parque Tornquist 6 y 7), donde el avance de la cubierta vegetal obstaculizaba su visualización en el terreno. 


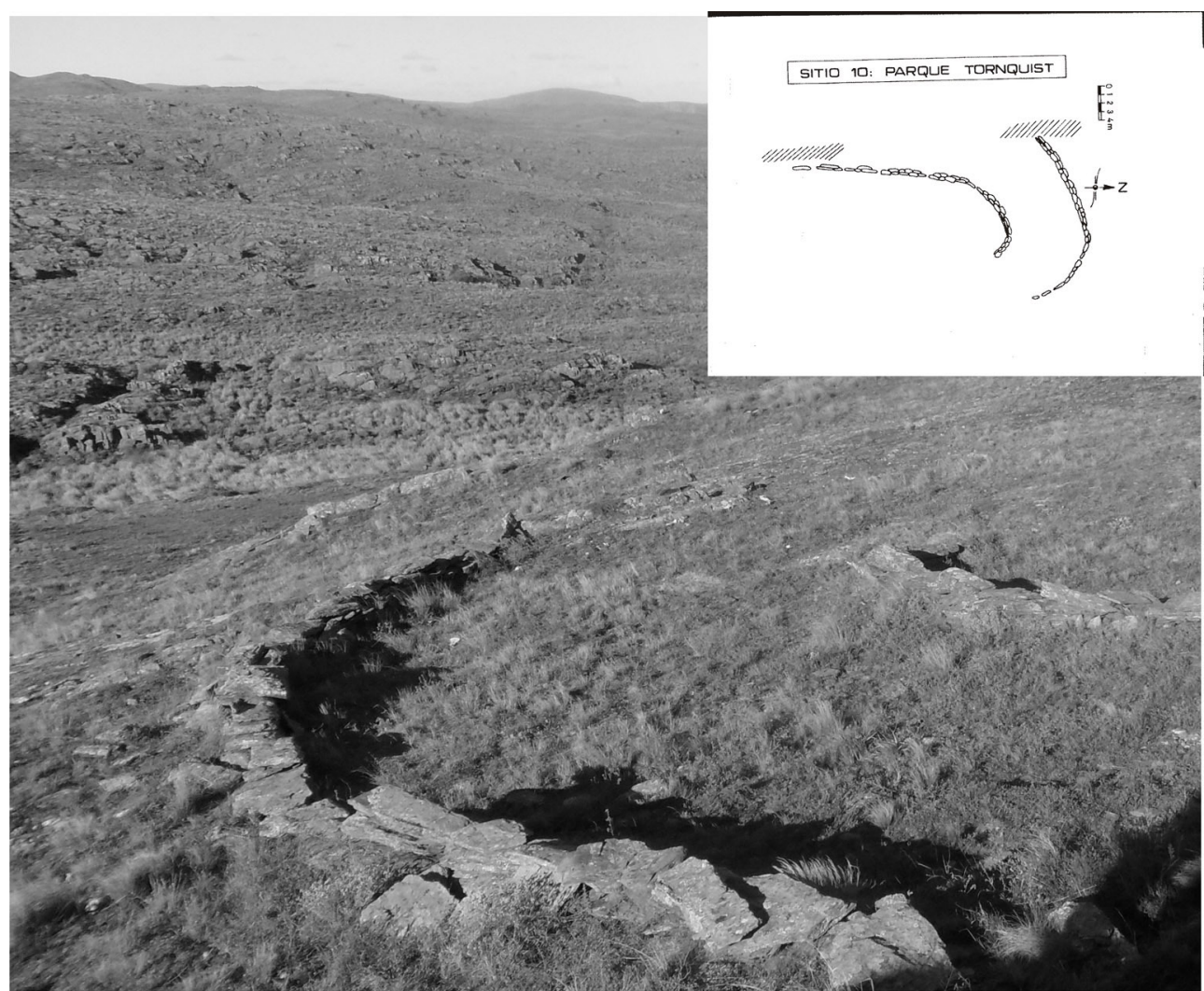

Figura 4. Dos pircados paralelos con forma subcircular en el sitio 10 del Parque Provincial E. Tornquist.

\section{Consideraciones Finales}

En este trabajo se han presentado nuevos relevamientos efectuados en sitios localizados en las nacientes del Sauce Grande con estructuras de piedras y piedras paradas. De los casos estudiados la mayoría se encuentra a menos de $100 \mathrm{~m}$ de un curso de agua (10 casos $=$ $77 \%$ ), y se encuentran en condiciones de visibilidad diferencial debido a la acción antrópica contemporánea (ie vandalismo directo sobre las estructuras de piedras, presencia de bosque de especies alóctonas, impacto de tareas agrícolas ganaderas, entre otros) lo cual dificulta su documentación, análisis y preservación. Sin embargo, debe mencionarse que las estructuras de piedra presentadas en este trabajo constituyen parte de un repertorio regional mayor (ver tabla en Oliva y Panizza 2012, y en Oliva 2011) con características distintivas. En este sentido, se observa (Tabla 1) que en general las estructuras se encuentran próximas a cursos de agua, no superando la distancia de $200 \mathrm{~m}$. Por otra parte, en cuanto a la técnica de construcción existe variabilidad de registro ya que en uno de los casos (PT 10) se encuentran pircados, mientras que en los restantes son combinaciones de piedras paradas o/y acumuladas. Cada uno de los sitios está compuesto de un tipo de elemento constitutivo (piedras paradas, acumuladas) excepto el caso de Las Vertientes 1 que se observa tanto piedras paradas como acumuladas. En general están compuestas por un tipo de elemento considerando que en el caso de los recintos se toma como unidad la estructura y en los alineamientos de piedras paradas se toma cada piedra como unidad. 
Los casos aquí presentados, localizados en las nacientes del río Sauce Grande en un área de unos $36 \mathrm{~km}^{2}$, reflejan por un lado parte de una alta concentración de sitios y al mismo tiempo se evidencia una variedad importante de los mismos. En tal sentido se observa que en las tres localidades analizadas existen estructuras aunque el patrón presentado difiere en cada caso. Particularmente en el sitio 1 de la localidad Las Vertientes la estructura presente es de reducidas medidas, unos $12 \mathrm{~m}^{2}$, en forma de dos " $\mathrm{u}$ " adosadas. Por su parte en el sitio 1 de la Localidad Las Acacias sus medidas son considerablemente mayores ya que el espacio comprendido es de unos $513 \mathrm{~m}^{2}$, de forma trapezoidal. Finalmente el sitio 10 del Parque Tornquist está integrado por dos estructuras de pircado con forma subcircular, paralelas entre sí, con un largo de $32 \mathrm{~m}$ de largo por $19 \mathrm{~m}$ de ancho, conformando una curva de $7 \mathrm{~m}$ de ancho por unos $18 \mathrm{~m}$ de largo. Un dato significativo es que el pircado interior se continúa en línea recta hacia el sur, siguiendo por unos $18 \mathrm{~m}$ más. Como se ha expresado en anteriores oportunidades (Oliva y Panizza 2012), se considera que el registro arqueológico de estructuras de piedra y piedras paradas sería producto de diversas actividades de las sociedades que habitaron el espacio serrano de Ventania al menos en el momento final del Holoceno. En la interpretación de este particular registro arqueológico de la Región Pampeana, limitado a los ambientes serranos, se consideró útil la aproximación teórica de la arqueología monumental como concepto clave para rediscutir las posibles asignaciones funcionales de este tipo de registro (Thomas 2001, García Sanjuán 2005, Criado y Villoch 1998). En determinados sectores de las regiones de Pampa y Patagonia, diferentes autores han abordado el tema de las "piedras paradas", "estructuras de piedras", "pilas de rocas", con grados disímiles de resolución (i.e. Goñi 1986-1987, 1988, 1991; Madrid 1991 a y b, Ramos 1995, 2001; Ramos et al. 2008, Borrero et al. 2011, Bognanni 2012) asumiendo diversas interpretaciones funcionales con respecto al registro presentado. Si bien escapa la discusión de las mismas al objetivo central de este trabajo, cabe destacar que en muchos de los casos se vincula a un circuito mayor de traslado de ganado (i.e. Goñi 1986-1987, Ramos 2001) o bien atribuibles a la construcción de chenques, pseudo-chenques o parapetos, entre otros (Borrero et al. 2011).

Con respecto al potencial de los análisis liquenométricos para establecer la edad mínima de las estructuras, cabe señalar que en regiones de alta amplitud térmica como son el noroeste argentino, la zona cordillerana o el sur del país (i.e. Borrero et al. 2011), se han aplicado estas técnicas de estimaciones cronológicas obteniendo resultados con cierto grado de confiabilidad. Sin embargo, esta situación no presenta las mismas posibilidades para la región Pampeana, en cuanto a las condiciones ambientales que dificultan la datación precisa de las estructuras, dado el rápido crecimiento de las diferentes especies, aunque su futura aplicación no se descarta, debido a los avances realizados por Lavornia et al. (2009) en vinculación con estudios de biomonitoreo, en base a la identificación de 26 especies de líquenes en las serranías del Sistema de Tandilia.

La información presentada en este trabajo se considera que aporta a la constitución de una base de sitios vinculada con las nacientes del río Sauce Grande, debida a una utilización estratégica de las cabeceras del río mediante la señalización a través de la creación de determinados hitos monumentales en el terreno, involucrando cuestiones tanto socioeconómicas como sociales-simbólicas, en donde el manejo territorial de los valles en relación con los cursos de agua juega un rol sustancial para los grupos cazadores recolectores del Sistema de Ventania. 


\section{Bibliografía citada}

Bognanni, F.

2012 Un estudio acerca del uso del espacio en arqueología de sitios históricos. "Corrales de indios" y rastrilladas: un análisis inter-regional. Tesis doctoral. Facultad de Ciencias Naturales y Museo, Universidad Nacional de La Plata.

Borrero, L. A., Borrazzo, K. B., Garibotti, I., y M. C.Pallo

2011 Concentraciones de pilas de rocas en la cuenca superior del río Santa Cruz (Argentina). Magallania [online]. vol.39, n.2, pp. 193-206. [citado 2013-03-11]

Criado, F. y V. Villoch

1998 La monumentalización del paisaje: percepción y sentido original en el megalitismo de la sierra de Barbazo (Galicia). Trabajos de Prehistoria 55 (1): 63-80.

Devoto, G.; C. Oliva y A. Sfeir

2012 Distribución del Registro Monumental presente en el Sistema Serrano de Ventania y su relación con las poblaciones actuales. Libro de Resúmenes del Simposio "Paisajes Arqueológicos del Holoceno Tardío. Interacciones entre seres humanos y entornos." Pp. 16. Rosario.

García Sanjuan, L.

2005 Las piedras de la memoria. La permanencia del megalitismo en el suroeste de la península ibérica durante el II y I milenios ANE. Trabajos de Prehistoria 62, nº 1, pp. 85 a 109.

Goñi, R. A.

1986-1987 Arqueología de sitios tardíos en el valle del río Malleo, Pcia. del Neuquén. Relaciones de la Sociedad Argentina de Antropología, vol. XVII, $\mathrm{n}^{\circ}$ 1:37-66.

1988 Arqueología de sitios tardíos en el Valle del Arroyo Quilca (Pcia. del Neuquén, Argentina). Resúmenes de ponencias científcas del IX Congreso Nacional de Arqueología Argentina. Buenos Aires. 1991 Arqueología de sitios tardíos en el valle del arroyo Vilcunco (Pcia. del Neuquén, Argentina). En Actas del XI Congreso Nacional de Arqueología Chilena. Santiago de Chile, pp.: 217-223.

Harrington, H. J.

1947 Explicación de las Hojas Geológicas 33m (Sierra de Curamalal) y 34m (Sierra La Ventana). Provincia de Buenos Aires. Boletín de la Dirección de Minería y Geología, 61. Buenos Aires.

Lavornia, J, M. Kristensen y V. Rosato

2009 Los líquenes de los roquedales de Tandil. Biondicadores de la calidad del aire. En Cinea. Estudios Ambientales 3: Área de Tandilia: 87-94.

Madrid, $P$.

1991a Estudio arqueológico de los sitios con estructuras de piedra en las sierras de Pillahuincó, provincia de Buenos Aires. Rev. del Museo de Historia Natural de San Rafael, Mendoza XI(3): 129-155.

1991b Infraestructura indígena para el mantenimiento y traslado de ganado introducido: el caso del Sistema Serrano de Pillahuinco, provincia de Buenos Aires. Boletín del Centro 3: 65-71. La Plata. 
Oliva, F.

2011 Estructuras de piedras del Sistema Serrano de Ventania. Evaluación de interpretaciones alternativas. Libro de resúmenes del IV Congreso Argentino de Arqueometría. Luján, 8 al 11 de noviembre de 2011. Pp. 34-35.

Oliva, F. y M. C. Panizza

2012 Primera Aproximación a la Arqueología Monumental del Sistema Serrano de Ventania, Provincia de Buenos Aires. Anuario de Arqueología 4: 161-180. Departamento de Arqueología. Escuela de Antropología - Facultad de Humanidades y Artes. Universidad Nacional de Rosario. Rosario.

2010 Arqueología monumental del Sistema Serrano de Ventania, provincia de Buenos Aires. Libro de resúmenes de las 3ras Jornadas Rosarinas de Arqueología. Rosario, 18 y 19 de noviembre de 2010. Pp. 15-16.

Oliva, F. y M. Roa

1998 Las estructuras de piedra del área serrana de Ventania, provincia de Buenos Aires. Presentado en las Primeras Jornadas de Arqueología Histórica de la Provincia y Ciudad de Buenos Aires. Quilmes.

Ramos, M.

1995 ¿Corrales o estructuras? Historical Archaeology in Latin America 15: 63-69.

2001 Arqueología de las construcciones líticas de Tandilia. En Arqueología uruguaya hacia el fin del milenio,Tomo II, pp. 579-587. Asociación Uruguaya de Arqueología, Colonia.

Ramos, M., F. Bognanni, M. Lanza, V. Helfer, P. Salatino, C. Quiroga, D. Aguirre y D. Pau 2008 "Corrales de indios" (Lithic Structures) in Tandilia, Argentina: a global study. International Journal for Historical Archaeology 12 (3): 209-247.

Roa, M. y M. Saghessi

2004. Estructuras de piedra en la cuenca del arroyo San Diego, partido de Tornquist. En $L a$ Región Pampeana - su pasado arqueológico-, C. J. Gradín y F. Oliva (eds.), pp. 175-188. Laborde

Sfeir, A. y F. Oliva

2012. De la extensión a la investigación. Arqueología monumental del Sistema Serrano de Ventania, provincia de Buenos Aires. REVISTA ExT , Vol 3, No 2: Resúmenes del 5to Congreso Nacional de Extension Universitaria. http:/ / revistas.unc.edu.ar/index.php/ext/ article/view/1474

Thomas, J.

2001. Arqueologías de lugar y paisaje. Archaeological Theory Today. Hodder, I. (Ed.). Pp. 165-186. Cambridge Polity. 